\title{
KINETIC ENERGIES IN THE DIRECT DOUBLE PHOTOIONIZATION OF HELIUM AND THE ELECTRON-ELECTRON CORRELATION
}

\author{
J. BAUER \\ Katedra Fizyki Jądrowej i Bezpieczeństwa Radiacyjnego \\ Uniwersytetu Łódzkiego, Pomorska 149/153, 90-236 Łódź, Poland
}

(Received October 28, 1999)

\begin{abstract}
We present a simple model describing kinetic energy probability distributions of outgoing electrons in the direct double photoionization of helium. The model applies to photons of energy greater than the binding energy of the atom and to radiation intensities extending from perturbation regime to the strong-field one. It appears that the shape of probability distribution is mainly determined by the electron-electron correlation in their final state. For nonperturbative intensities the distribution depends strongly on polarization of incident radiation.
\end{abstract}

PACS numbers: $32.80 . \mathrm{Rm}, 33.80 . \mathrm{Rv}$

\section{Introduction}

In a recent paper Dörner et al. [1] have reported an interesting experiment in which fully differential cross-sections, for the double photoionization of helium by a synchrotron (linearly polarized) photons of energies 1-80 eV above threshold, have been measured. This means that the final state of the system has been kinematically completely determined, except for electron spins. For every double ionization event, the momenta of both escaping electrons and $\mathrm{He}^{2+}$ recoil ion have been known in a complete momentum space ( $4 \pi$ solid angle). It appears that the use of collective momentum coordinates for two electrons reveals simple features of the correlated motion of these electrons. These momenta are the center-of-mass (CM) motion momentum $\boldsymbol{P}=p_{1}+p_{2}$ and the relative motion one $p=\left(p_{1}-p_{2}\right) / 2$, where $p_{1}$ and $p_{2}$ refer to single electrons.

The same collective momenta with canonically conjugate spatial coordinates ( $\boldsymbol{R}=\left(\boldsymbol{r}_{1}+r_{2}\right) / 2$ and $r=r_{1}-r_{2}$ respectively) have been applied by us [2] to describe a direct (nonsequential) double ionization of helium by a strong circularly polarized plane wave (the notation in [1] is opposite to the notation here, which is 
the same as in [2]). We have generalized [2] the nonrelativistic strong-field approximation (SFA), the Reiss version of the Keldysh-Faisal-Reiss (KFR) models [3-5] to the case of a two-electron atom. The SFA is supposed to be valid for a very intense field, when the average oscillation energy of the detached electron in the laser field (ponderomotive potential) dominates the atomic binding energy [5]. The essence of the SFA is that it neglects the nucleus Coulomb influence on the final state of ionized electron. As the laser field becomes stronger, the SFA improves and it is particularly suited in a stabilization regime, where the ionization rate is a decreasing function of radiation intensity.

Nevertheless, if the photon energy is greater than the electron binding energy $\left(\omega>E_{\mathrm{B}}\right.$ in atomic units (a.u.), which are used throughout the paper: $\hbar=e=m_{\mathrm{e}}=1$ ), the one-electron SFA in the low-intensity limit reduces to the ordinary nonrelativistic first-order perturbation theory result [5]. In this limit ionization is only one-photon, its rate is proportional to radiation intensity, and an asymptotic momentum of outgoing electron is given by the energy conservation law: $p^{2} / 2=\omega-E_{\mathrm{B}}$. There are two simplifications in this perturbation theory. The first one is often applied in atomic physics, because this is the dipole approximation for the electromagnetic field (i.e. it depends only on time, if $\omega \ll c \approx 137$ a.u.). In the final state of outgoing electron the Coulomb wave function of unbound (positive-energy) electron is replaced by a plane wave. This is the other simplification, which is justifiable for photon energy not very close to the threshold. If one makes the same perturbation calculation without the plane-wave approximation of the ionized electron, one obtains the same result for the ionization rate (differential or total) up to some constant factor [6]. This factor depends only on $\omega$ and $E_{\mathrm{B}}$. Thus, under the above mentioned conditions, the Coulomb attraction between nucleus and ionized electron has no influence on relative (divided by the total ionization rate) spatial probability distribution of the electron.

Also the low-intensity limit of the two-electron SFA is equivalent to perturbation calculation with $H_{\mathrm{I}}=\boldsymbol{P} \boldsymbol{A}(t) / c$ (the atom-field interaction Hamiltonian), and a final state of two outgoing electrons, which is a product of the plane wave in the $\mathrm{CM}$ motion and the repulsive Coulomb wave function in the relative motion (see next section). In this final state of electron pair its interaction with the nucleus is neglected. Thus the total ionization rate usually differs from the true result. But by the analogy with the one-electron case we may at least expect that such a simple model should qualitatively well describe the spatial distributions of ionized electrons. This has been demonstrated by Becker and Faisal [7], who compared the triply differential cross-sections calculated from the correlated KFR theory (which is in fact the same as the two-electron SFA [2]) with the experimental data of Schwarzkopf et al. [8].

Theoreticians put a considerable effort into the detailed calculations for the $(\gamma, 2 \mathrm{e})$ on helium [9] (it is impossible to give all references and here we quote some recent articles only), because this is probably the simplest many-electron process. The purpose of this paper is not to improve such theories, but to show that some features of one-photon two-electron weak-field ionization may be described in a very simple way, which can be applied also to description of the strong-field ionization. 


\section{Direct double ionization in the SFA}

In the dipole approximation, in the final state (when we neglect the nucleus of helium atom) both electrons respond to the laser field identically and oscillate in phase. As a result, the motion can be separated into the oscillations of the center-of-mass in the laser field and field-free Coulomb motion of the relative coordinate [10]. Thus the SFA probability amplitude of direct (nonsequential) double ionization is given by the following expression [2]:

$$
\begin{aligned}
& (S-1)_{\mathrm{fi}} \\
& \quad=-\mathrm{i} \int_{-\infty}^{+\infty} \mathrm{d} t \int \mathrm{d}^{3} R \int \mathrm{d}^{3} r\left[\Psi_{\boldsymbol{P}}^{V}(\boldsymbol{R}, t) \Phi_{\boldsymbol{p}}^{(-)}(r, t)\right]^{*} H_{\mathrm{I}}(\boldsymbol{P}, t) \Phi_{\mathrm{i}}(\boldsymbol{R}, r, t),
\end{aligned}
$$

where the laser-atom interaction Hamiltonian is given by

$$
H_{\mathrm{I}}(\boldsymbol{P}, t)=\frac{1}{c} \boldsymbol{A}(t) \boldsymbol{P}+\frac{1}{c^{2}} \boldsymbol{A}(t)^{2}
$$

$\left(\boldsymbol{P}=p_{1}+p_{2}\right.$ is the CM motion momentum operator in Eq. (2)). The final state of two ionized electrons is the product of the Volkov-type wave function of the CM motion

$$
\Psi_{\boldsymbol{P}}^{\mathrm{V}}(\boldsymbol{R}, t)=\exp \left[\mathrm{i} \boldsymbol{P} \boldsymbol{R}-\mathrm{i} \frac{P^{2}}{4} t-\mathrm{i} \int_{-\infty}^{t} \mathrm{~d} \tau H_{\mathrm{I}}(\boldsymbol{P}, \tau)\right],
$$

and field-free wave function in the repulsive Coulomb potential of the relative motion $\left(p=\left(p_{1}-p_{2}\right) / 2, \Gamma\right.$ is the Euler gamma function, $F$ is the confluent hypergeometric function in Eq. (4))

$$
\begin{aligned}
\Phi_{p}^{(-)}(r, t) & \equiv \Phi_{p}^{(-)}(r) \exp \left(-\mathrm{i} p^{2} t\right)=\Gamma\left(1-\frac{\mathrm{i}}{2 p}\right) \\
& \times \exp \left(-\frac{\pi}{4 p}+\mathrm{i} p r\right) F\left(\frac{\mathrm{i}}{2 p}, 1,-\mathrm{i}(p r+p r)\right) \exp \left(-\mathrm{i} p^{2} t\right) .
\end{aligned}
$$

$\Phi_{\mathrm{i}}(\boldsymbol{R}, \boldsymbol{r}, t)$ should be the exact ground state of helium (the initial state). We approximate it by the wave function including only the electron-electron (e-e) radial correlation. This function has been constructed in a way similar to the (less accurate) one before [2, Appendix B], on the ground of the following wave function:

$$
\Phi_{\mathrm{i}}\left(r_{1}, r_{2}, t\right)=N \exp \left(\mathrm{i} E_{\mathrm{B}} t\right)\left[\exp \left(-\alpha r_{1}-\beta r_{2}\right)+\exp \left(-\alpha r_{2}-\beta r_{1}\right)\right]
$$

with $\alpha=2.179, \beta=1.189, E_{\mathrm{B}}=2.904$ (the experimental value in a.u.), $N=0.7070$. Our initial-state wave function assumes the shape of $\Phi_{\mathrm{i}}(R, r, t)$, where $R=|\boldsymbol{R}|, r=|r|$, because it considerably reduces numerical difficulty of the problem. The overlap of this function with the wave function (5) is 0.964 . The probability of nonsequential double ionization per unit time, $w$, is found from

$$
w=\lim _{t \rightarrow \infty} \frac{1}{t}\left|(S-1)_{\mathrm{fi}}\right|^{2} .
$$

The result for circularly polarized radiation is

$$
w=2 \pi \omega^{2}|G(P, p)|^{2} \sum_{n=-\infty}^{\infty}(n-2 z)^{2} J_{n}^{2}\left(\sqrt{\frac{2 z}{\omega}} P \sin \vartheta_{P}\right)
$$




$$
\times \delta\left[E_{\mathrm{B}}+P^{2} / 4+p^{2}+(2 z-n) \omega\right] .
$$

For linearly polarized radiation one has to replace the ordinary Bessel function by the generalized two-argument one [5]

$$
J_{n}^{2}\left(\sqrt{\frac{2 z}{\omega}} P \sin \vartheta_{P}\right) \rightarrow J_{n}^{2}\left(2 \sqrt{\frac{z}{\omega}} P \cos \vartheta_{P},-z\right) .
$$

Expressions $(7,8)$ describe differential transition rates to the unbound two-electron final state, which is parametrized by $\boldsymbol{P}$ and $p$ vectors. One can find the details of calculations leading to Eq. (7) in our paper [2]. In the above expressions there are: $z$ - the intensity parameter equal to the ponderomotive potential divided by the frequency (intensity $\left.=4 \omega^{3} z\right) ; \vartheta_{P}-$ the polar angle of $\boldsymbol{P}(z$-axis is in the direction of incoming radiation for circular polarization, and in the direction of polarization vector - for linear polarization); and

$$
G(P, p) \equiv \int \mathrm{d}^{3} r\left(\Phi_{p}^{(-)}(r)\right)^{*} \int \mathrm{d}^{3} R \exp (-\mathrm{i} \boldsymbol{P R}) \Phi_{\mathrm{i}}(R, r) .
$$

An infinitesimal volume element in the six-dimensional space of two-electron final-states is equal to $\mathrm{d}^{3} P \mathrm{~d}^{3} p /(2 \pi)^{6}=2 \mathrm{~d} \Omega_{P} \mathrm{~d} \Omega_{p} \sqrt{E_{R} E_{r}} \mathrm{~d} E_{R} \mathrm{~d} E_{r} /(2 \pi)^{6}$, where

$$
E_{R}=P^{2} / 4, \quad E_{r}=p^{2}
$$

are the $\mathrm{CM}$ and relative motion kinetic energies. Dividing this volume element by $\mathrm{d} E_{R}$ and $\mathrm{d} E_{r}$ respectively, we get two different five-dimensional elements. Integrating separately ionization rate (7), with the substitution (8), over these elements in the entire space, we obtain

$$
\begin{aligned}
\frac{\mathrm{d} W}{\mathrm{~d} E_{R}} & =\frac{\omega^{2}}{\pi^{3}} \sum_{n=n_{0}\left(E_{R}\right)}^{\infty}(n-2 z)^{2} \sqrt{E_{R}\left(E_{n}-E_{R}\right)}\left[G\left(P\left(E_{R}\right), p\left(E_{n}-E_{R}\right)\right)\right]^{2} \\
& \times \int_{0}^{\pi / 2} \mathrm{~d} \vartheta_{P} \sin \vartheta_{P} J_{n}^{2}\left(4 \sqrt{\frac{z E_{R}}{\omega}} \cos \vartheta_{P},-z\right), \\
\frac{\mathrm{d} W}{\mathrm{~d} E_{r}} & =\frac{\omega^{2}}{\pi^{3}} \sum_{n=n_{0}\left(E_{r}\right)}^{\infty}(n-2 z)^{2} \sqrt{\left(E_{n}-E_{r}\right) E_{r}}\left[G\left(P\left(E_{n}-E_{r}\right), p\left(E_{r}\right)\right)\right]^{2} \\
& \times \int_{0}^{\pi / 2} \mathrm{~d} \vartheta_{P} \sin \vartheta_{P} J_{n}^{2}\left(4 \sqrt{\frac{z\left(E_{n}-E_{r}\right)}{\omega}} \cos \vartheta_{P},-z\right) .
\end{aligned}
$$

These are the total direct double ionization rates per unit of kinetic energy $\left(0 \leq E_{R}, E_{r}<\infty\right)$ for linear polarization of incoming radiation. In Eqs. (11) the momenta are calculated from relation inverse to (10). The minimal values of indices over which we sum depend on energy: $n_{0}\left(E_{R}\right)=\left[2 z+\left(E_{\mathrm{B}}+E_{R}\right) / \omega\right]+1, n_{0}\left(E_{r}\right)=$ $\left[2 z+\left(E_{\mathrm{B}}+E_{r}\right) / \omega\right]+1([x]$ means integer part of the real number $x)$. Each of expression (11) is an infinite sum of terms describing different $n$-photon ionization channels. Not all the channels, opened at a given intensity, contribute to the series (11). Of course, only these $n$-photon processes are included, in which the total 
kinetic energy of two outgoing electrons is greater than their given kinetic energy of $\mathrm{CM}$ or relative motion respectively $\left(E_{n}=(n-2 z) \omega-E_{\mathrm{B}}>E_{R}\right.$ or $\left.E_{r}\right)$.

In the low-intensity limit $(z \rightarrow 0)$ ionization rate per unit energy simplifies considerably. The leading term of the generalized Bessel function $J_{n}(\sqrt{z} \mu, z \nu)$ is proportional to $z^{n / 2}$ for small $z$ [5], so the dominant terms in the series (11) have the numbers $n=n_{0}\left(E_{R}\right)$ and $n=n_{0}\left(E_{r}\right)$. Since $z \rightarrow 0$ and $\omega>E_{\mathrm{B}}$, then $n_{0}\left(E_{R}\right)=n_{0}\left(E_{r}\right)=1$ for kinetic energies fulfilling the condition: $0 \leq E_{R}, E_{r}<$ $\omega-E_{\mathrm{B}}$. Energies from this range stand for the one-photon ionization. Greater energies correspond to many-photon processes, for which $n_{0}\left(E_{R}\right), n_{0}\left(E_{r}\right) \geq 2$. Thus, in the limit discussed here, these processes are much weaker and one can neglect them. Replacing in Eqs. (11) the Bessel functions with its leading terms for $z \rightarrow 0: J_{1}(\sqrt{z} \mu, z \nu) \approx \sqrt{z \mu^{2}} / 2$, we can easily integrate over $\vartheta_{P}$ and obtain

$$
\begin{aligned}
& \frac{\mathrm{d} W}{\mathrm{~d} E_{R}}=\frac{\omega z}{3 \pi^{3}} E_{R} \sqrt{E_{R}\left(E-E_{R}\right)}\left[G\left(P\left(E_{R}\right), p\left(E-E_{R}\right)\right)\right]^{2}, \\
& \frac{\mathrm{d} W}{\mathrm{~d} E_{r}}=\frac{\omega z}{3 \pi^{3}}\left(E-E_{r}\right) \sqrt{\left(E-E_{r}\right) E_{r}}\left[G\left(P\left(E-E_{r}\right),\left(E_{r}\right)\right)\right]^{2} .
\end{aligned}
$$

The low-intensity result (12) holds true also for circular polarization. $E=\omega-E_{\mathrm{B}}$ is the photon excess energy. Due to the energy conservation law we have: $E=$ $E_{R}+E_{r}$. The function $G(P, p)$ may include the e-e repulsion in the final state of ionized electrons or not. In the latter case one has to replace $\Phi_{p}^{(-)}(r)$ by an ordinary plane wave in Eq. (9). Of course, expressions (12a), (12b), which are the functions of $E_{R}$ and $E_{r}$ respectively, when integrated in the interval $[0, E]$ must give the total two-electron ionization rate $W$. The total cross-section $\sigma^{++}$ for discussed process is equal to $W$ divided by the photon flux $\left(c \omega^{2} z /(2 \pi)\right.$ in a.u.).

\section{Results and discussion}

In Fig. 1 we show cross-sections differential in energy (total cross-sections per unit of kinetic energy of outgoing electrons) for direct double photoionization of helium. $\mathrm{d} \sigma^{++} / \mathrm{d} E_{R}$ and $\mathrm{d} \sigma^{++} / \mathrm{d} E_{r}$ are plotted as functions of $E_{R} / E$ and $E_{r} / E$ respectively (the fraction of photon excess energy $E$ ). There are four different excess energies $E$ here: 1, 6, 20,80 eV. The full (CM motion) and the open (relative motion) circles stand for the experimental data of Dörner et al. [1]. The solid (CM motion) and the dashed (relative motion) lines are our results (12), which have been normalized on an absolute scale to fit the maximum.

The axial symmetry in Fig. 1 is a consequence of equation: $E=E_{R}+E_{r}$. If one finds an electron pair with the kinetic energy of CM motion around some $E_{R}$, it means that its relative motion kinetic energy is around $E_{r}=E-E_{R}$. The agreement between our model and the experiment is quite good (except for $E=1 \mathrm{eV}$, where our plane-wave approximation for the CM motion should not work well). It may be well to add that there exist much more complicated [9] theories, which better describe the regularities observed in the weak-field experiments. However, it is the advantage of the SFA that enables us to study nonsequential double ionization also for superintense laser fields.

The cross-section differential in energy $\left(\mathrm{d} \sigma^{++} / \mathrm{d} E_{R}\right.$ or $\left.\mathrm{d} \sigma^{++} / \mathrm{d} E_{r}\right)$ as a function of kinetic energy ( $E_{R}$ or $E_{r}$ respectively) considerably changes its shape and 

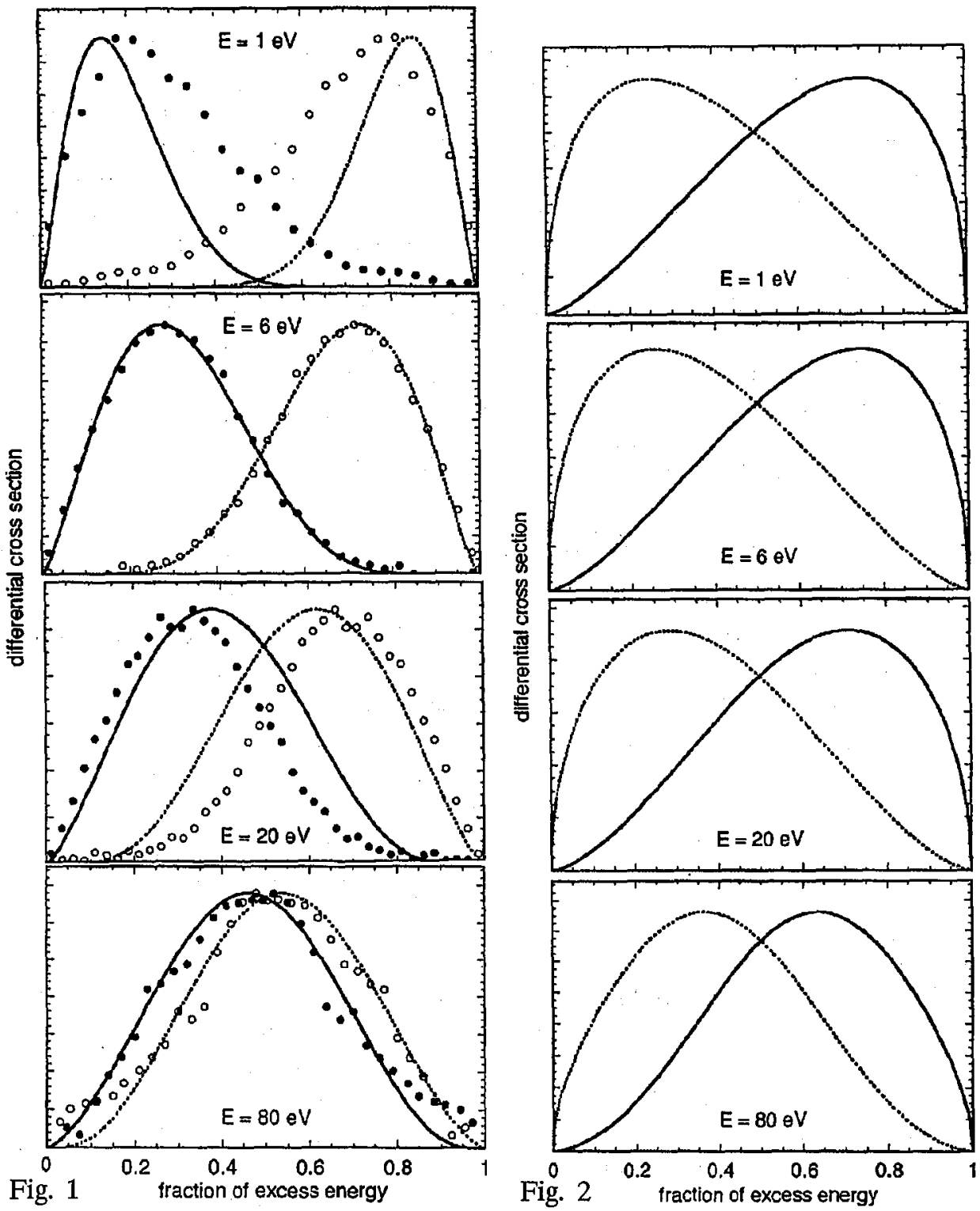

Fig. 1. Cross-sections differential in energy (in arbitrary units) plotted as a function of the fraction of the excess energy $\left(E=\hbar \omega-E_{\mathrm{B}}\right)$ in the CM motion (full circles, solid lines) and the relative motion (open circles, dashed lines). The circles are the experimental data from Ref. [1], and the lines show the low-intensity limit of the two-electron SFA (with the final state e-e correlation included). From top to bottom the separate graphs correspond to increasing excess energies: $1,6,20$, and $80 \mathrm{eV}$.

Fig. 2. Cross-sections differential in energy (in arbitrary units) plotted as a function of the fraction of the excess energy in the CM motion (solid lines) and the relative motion (dashed lines). The low-intensity limit of the two-electron SFA is utilized here, but without the final state e-e correlation. From top to bottom the separate graphs correspond to increasing excess energies: $1,6,20$, and $80 \mathrm{eV}$. 
the position of the maximum if we neglect the Coulomb repulsion in the final state of two outgoing electrons. In Fig. 2 there are the same probability distributions as in Fig. 1, but without the final state e-e correlation. When the photon excess energy $E$ increases, both distributions (with and without the e-e correlation) become more similar, so the role of Coulomb repulsion vanishes. Close to the threshold much more kinetic energy falls to the relative motion than to the $\mathrm{CM}$ one (Fig. 1). With growing $E$ the shares of both types of kinetic energy make even, but even for $E=80 \mathrm{eV}$ somewhat more energy falls to the relative motion. On the other hand, neglecting the e-e correlation we get the opposite relation more energy falls to the CM motion.

The total ionization rate is proportional to the radiation intensity if it is weak enough. This situation is connected with one-photon ionization, which is well described by perturbation calculation for the atom in an electromagnetic field. When intensity increases, the ponderomotive potential (average kinetic energy of oscillations) of ionized electrons becomes comparable with their binding energy in the helium atom. Then we approach the proper applicability range of the SFA. The more and more probable becomes the process consisting in two-photon absorption. The ionization rate usually grows slower than linearly, and near some value of intensity it can rapidly go down (particularly for linear polarization), which is the sign of photon threshold. It means that due to the increase in ponderomotive potential the one-photon channel of ionization gets closed. Nowadays, for photons of energy greater than $79 \mathrm{eV}$, the maximum experimentally attainable intensities are much smaller than those for which above-threshold ionization (ATI) could come out. But this situation may change in a few years. A free-electron laser source at DESY laboratory is expected to produce an intense laser beam with photon energy up to $30 \mathrm{eV}$ and intensity of the order of one atomic unit $\left(I_{\text {at }} \approx 3.51 \times 10^{16} \mathrm{~W} / \mathrm{cm}^{2}\right)$. The He atom with its binding energy of $79 \mathrm{eV}$ could be replaced by an atom with two valence electrons like $\mathrm{Ca}$ or $\mathrm{Mg}$, for whose the binding energy of both outer electrons is much smaller $(12 \mathrm{eV}$ and $15 \mathrm{eV}$ respectively [11]).

Applying the SFA one can answer the following question: for what intensity and in what way would the probability distributions (measured in the experiment of the type [1]) change, if the two-photon ionization was comparable with the one-photon one. In Figs. 3 (linear polarization) and 4 (circular polarization) we show how the total ionization rate per unit of kinetic energy varies with increasing intensity for the photon excess energy $E=80 \mathrm{eV}$. In the dipole approximation both ionized electrons oscillate in phase. Due to their ponderomotive potential we observe a shift towards the smaller energies, gradual closing of the one-photon channel and emergence of another peak connected with two-photon ionization. This resembles a typical behavior of ATI peaks in a single electron ionization. The probability distributions change rapidly with increasing intensity, which has been chosen in such a way that from the top to the bottom of Figs. 3 and 4 the fraction of one-photon ionization is approximately equal to $90 \%, 50 \%, 10 \%$, and $1 \%$. The rest is connected mainly with two-photon ionization, but there are some very small contributions (growing with intensity) connected with the absorption of a greater number of photons. One can see that the shape of these energy distributions evolves 

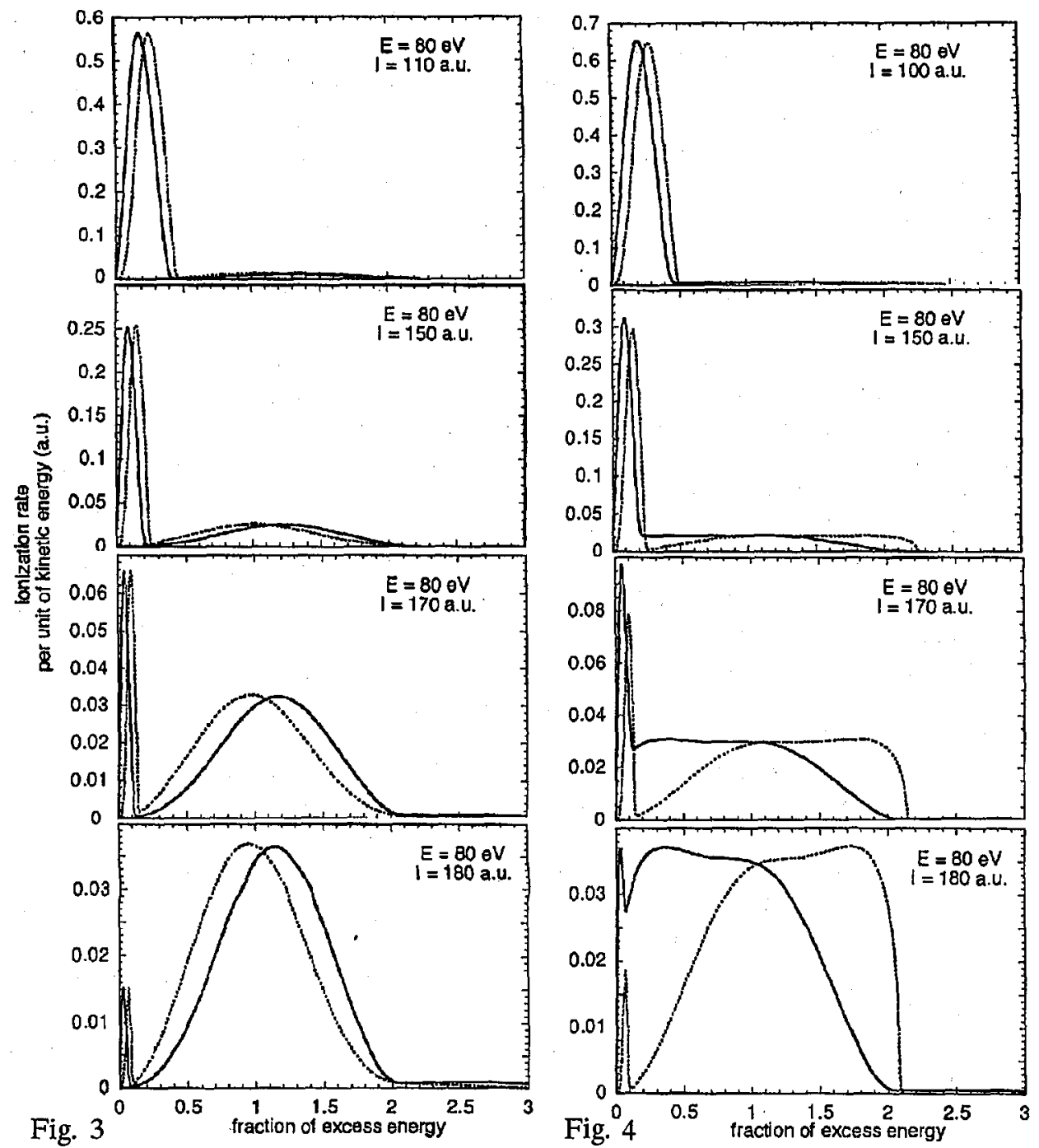

Fig. 3. Total ionization rates per unit of kinetic energy for the CM motion (solid lines) and the relative motion (dashed lines) in the two-electron SFA for circular polarization. The photon excess energy is $E=80 \mathrm{eV}$. From top to bottom the separate graphs correspond to increasing intensity $(I)$ and decreasing fraction of one-photon ionization: $90 \%, 50 \%, 10 \%$, and $1 \%$ (approximately).

Fig. 4. The same as in Fig. 3, but for linear polarization. From top to bottom intensity increases in such a way that the fraction of one-photon ionization is approximately equal to: $90 \%, 50 \%, 10 \%$, and $1 \%$.

(with growing intensity) in a very different way for both kinds of polarization. The interesting feature of two-photon ionization by circularly polarized plane wave is the change of order of the energetic maxima - the mean kinetic energy for the 
CM motion is greater than the mean kinetic energy for the relative motion of two outgoing electrons (Fig. 3). This is not observed for linear polarization (Fig. 4).

\section{Acknowledgments}

The author is indebted to Professor Kazimierz Rzązewski for his assistance and to Professor Reinhard Dörner for making accessible his experimental results. The paper has been supported by the grant No. 2 P03B 04710 of the Committee for Scientific Research.

\section{References}

[1] R. Dörner, J.M. Feagin, C.L. Cocke, H. Bräuning, O. Jagutzki, M. Jung, E.P. Kanter, H. Khemliche, S. Kravis, V. Mergel, M.H. Prior, H. Schmidt-Böcking, L. Spielberger, J. Ullrich, M. Unversagt, T. Vogt, Phys. Rev. Lett. 77, 1024 (1996).

[2] J. Bauer, K. Rzążewski, J. Phys. B 29, 3351 (1996).

[3] L.V. Keldysh, Zh. Eksp. Teor. Fiz. 47, 1945 (1964) [Sov. Phys.-JETP 20, 1307 (1965)].

[4] F.H.M. Faisal, J. Phys. B 6, L89 (1973).

[5] H.R. Reiss, Phys. Rev. A 22, 1786 (1980).

[6] K. Gottfried, Quantum Mechanics, Vol. I, Fundamentals, W.A. Benjamin Inc., New York 1966.

[7] A. Becker, F.H.M. Faisal, Phys. Rev. A 50, 3256 (1994).

[8] O. Schwarzkopf, B. Krässig, J. Elmiger, V. Schmidt, Phys. Rev. Lett. 70, 3008 (1993).

[9] S.P. Lucey, J. Rasch, C.T. Whelan, H.R.J. Walters, J. Phys. B 31, 1237 (1998); A.S. Kheifets, I. Bray, J. Phys. B 31, L447 (1998); J.M. Feagin, J. Phys. B 29, L551 (1996); M. Pont, R. Shakeshaft, Phys. Rev. A 54, 1448 (1996); F. Maulbetsch, M. Pont, J.S. Briggs, R. Shakeshaft, J. Phys. B 28, L341 (1995); A.K. Kazansky, V.N. Ostrovsky, J. Phys. B 28, 1453 (1995); F. Maulbetsch, J.S. Briggs, J. Phys. B 27, 4095 (1994).

[10] F.H.M. Faisal, Phys. Lett. A 187, 180 (1994).

[11] S. Bashkin, J.O. Stoner, Atomic Energy Levels and Grotrian Diagrams, North-Holland, Amsterdam 1975. 